\title{
Quantifying nanoparticle dispersion: application of the Delaunay network for objective analysis of sample micrographs
}

\author{
D. J. Bray • S. G. Gilmour · F. J. Guild • \\ T. H. Hsieh · K. Masania - A. C. Taylor
}

Received: 22 February 2011/ Accepted: 7 May 2011

(C) Springer Science+Business Media, LLC 2011

\begin{abstract}
Measuring quantitatively the nanoparticle dispersion of a composite material requires more than choosing a particular parameter and determining its correspondence to good and bad dispersion. It additionally requires anticipation of the measure's behaviour towards imperfect experimental data, such as that which can be obtained from a limited number of samples. It should be recognised that different samples from a common parent population can give statistically different responses due to sample variation alone and a measure of the likelihood of this occurring allows a decision on the dispersion to be made. It is also important to factor into the analysis the quality of the data in the micrograph with it: (a) being incomplete because some of the particles present in the micrograph are indistinguishable or go unseen; (b) including additional responses which are false. With the use of our preferred method, this article investigates the effects on the measured dispersion quality of nanoparticles of the micrograph's magnification settings, the role of the fraction of nanoparticles visible and the number of micrographs used. It is demonstrated that the best choice of magnification, which gives the clearest indication of dispersion type, is dependent on the type of nanoparticle structure
\end{abstract}

D. J. Bray (ه) - F. J. Guild · T. H. Hsieh · K. Masania ·

A. C. Taylor

Department of Mechanical Engineering, Imperial College London, South Kensington Campus, London SW7 2AZ, UK

e-mail: d.bray@imperial.ac.uk

D. J. Bray

School of Mathematical Science, Queen Mary University of London, Mile End Road, London E1 4NS, UK

S. G. Gilmour

Statistical Sciences Research Institute, University of Southampton, Highfield, Southampton SO17 1BJ, UK present. Furthermore, it is found that the measured dispersion can be modified by particle loss, through the limitations of micrograph construction, and material/ microscope imperfections such as cut marks and optical aberrations which could lead to the wrong conclusions being drawn. The article finishes by showing the versatility of the dispersion measure by characterising various different spatial features.

\section{Introduction}

Many studies into composite materials have shown that the dispersion quality of nanoparticles can have adverse effects on the material's mechanical performance. Given its importance concerning measurable properties, such as fracture toughness; fracture energy, strain; stiffness [1-7], it is no longer sufficient to simply state from viewing a micrograph that the nanoparticles are well- or poorly-dispersed. Instead exact knowledge of the extent of dispersion is sought such that correlations can be identified. This requires a robust quantitative measure for dispersion.

Dispersion has been variously defined, depending on requirement (for example, with respect to particle size distribution and orientations [8], homogeneity [9], regularity [10] etc.), but here it is taken to characterise how well the nanoparticles are spread through the whole material in terms of their locations. A well-dispersed system has a homogeneous spread of particles across the system, such that either the geometry of particles is completely random or better still the particles are equally spaced in a near lattice-like formation. Conversely, a poorly-dispersed system has particles which are clustered or heterogeneously dispersed such that the system is geometrically more disordered than would be expected if completely random. 
This geometric disorder provides an intuitive perspective on which to base a dispersion measure. Our study exploits the properties of the Delaunay network (the counterpart to Voronoi/Dirichlet tessellation which defines each Voronoi polygon as the region of the system in which a specific particle is found to be the nearest), that is generated using the positions of nanoparticles [9, 11-16]. Good reviews and alternative methods are also provided by [8, 17, 18, 19].

It is impractical to base the analysis on the complete system, given the difficulty of registering every particle in a material. The alternative is to suffice with a limited number of micrographs each showing a small local region of the material. Therefore, the challenge of developing a method is ensuring an objective assessment of dispersion is made when analysing data that is mathematically imperfect.

This article will develop the methodology to perform objective experimental assessment of the dispersion of nanoparticles in a material based upon sample micrographs. A quantity called the Area Disorder is used to determine the type of dispersion. The effects of varying the number, placement and magnification of the micrographs taken from any one material are investigated to check the robustness of the technique. Two questions will be of particular interest: (1) what is the extent of the dispersion? (2) how confident can we be that we have reached the correct conclusion about the material?

\section{The technique}

The technique employed in this article is simple to perform and exploits the unique property of lattice arranged particles in which triplets of nearest neighbours are regularly spaced apart. A detailed mathematical description has been provided in concurrent work [20, 21]. This article considers how it may be applied in a materials science context.

Each micrograph is made up of $n$ by $n$ pixels with a pixel length $l$, determined by the magnification used, such that each pixel covers an area $l^{2} \mathrm{~m}^{2}$ in the material (note this assumes that the micrographs are square, but the technique is readily adapted for rectangular micrographs). The total area of the material shown by the micrograph is $L^{2} \mathrm{~m}^{2}$ where $L=n l \mathrm{~m}$. Within the micrograph is a collection of $N$ identifiable nanoparticles (where $N$ must be larger than 3 ) with cross-sectional areas $a_{i} \mathrm{~m}^{2}, i$ being an integer between 1 and $N$, such that the area fraction $\left(A_{f}\right)$ of the micrograph covered by nanoparticles is $A_{f}=\sum_{i=1}^{N} a_{i} / L^{2}$.

For each particle the centre of mass point is found and these positions are used to generate a Delaunay network of triangular cells, making the assumption that the system obeys periodic boundary conditions. Each triangle has vertices that lie on the centre of mass points of a triplet of particles that are considered to be mutual nearest neighbours, whereby the associated Voronoi polygons of the three particles border one another to share a common vertex position. Periodic boundary conditions are used at the edges of the micrograph as these provide a convenient approach for generating a Delaunay network that spans the complete micrograph. This allows the following analysis to be based upon an assessment of the whole micrograph and not the smaller sub-region given by the hull of the particles. Figure 1 demonstrates the generation of the Delaunay network over the particle positions. Particles within the dashed edges of the rectangle belong to the micrograph, with the remainder being 'virtual' particles generated through the periodic boundary conditions. Only those Delaunay triangles (all triangles of which have been outlined in Fig. 1) with centres that lie within the micrograph are used in the analysis. The resultant Delaunay network consists of $2 N$ triangles with areas $\Omega \mathrm{m}^{2}$, one of which is highlighted in Fig. 1. Although $N>3$ can be small, as shown here, the analysis is simplified when the number of particles is greater than 100 .

\section{Area Disorder}

The Area Disorder of the Delaunay network $\left(\mathrm{AD}_{\mathrm{Del}}\right)$ is a dimensionless quantity with values between 0 and 1 (having originally been defined for use with Voronoi tessellation in [22]). It is defined as

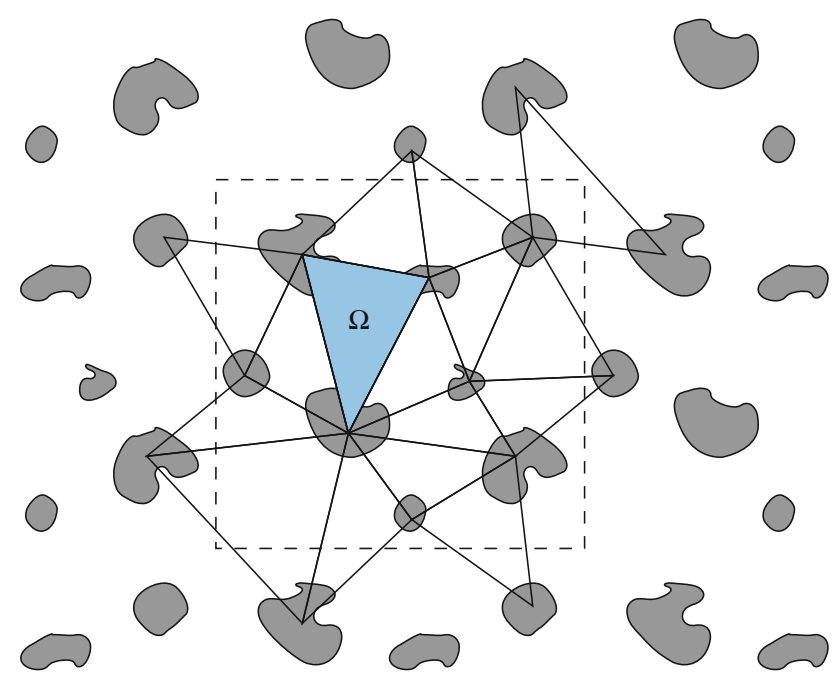

Fig. 1 Demonstration of generating Delaunay triangles from particle positions 
$\mathrm{AD}_{\text {Del }}=1-\left(1+s_{\Omega} / \bar{\Omega}\right)^{-1}$,

where $\bar{\Omega}$ and $s_{\Omega}$ are the mean and standard deviation of the Delaunay triangles' areas, respectively. Applying periodic boundary conditions reduces the Area Disorder to a single variable quantity of $s_{\Omega}$ with $\bar{\Omega}=L^{2} /(2 N)$ such that

$\mathrm{AD}_{\text {Del }}=1-\left(1+2 N s_{\Omega} / L^{2}\right)^{-1}$.

The type of dispersion present can be determined by calculating the mean value for the Area Disorder of a material, $\overline{\mathrm{AD}}_{\text {Del }}$. For example, an $\overline{\mathrm{AD}}_{\text {Del }}$ of zero implies a lattice arrangement of particles and hence a perfectly dispersed system. Similarly, a random arrangement of particles gives a mean Area Disorder of less than 0.478, with the exact value dependent upon the area fraction of particles within the micrograph. Larger values for $\overline{\mathrm{AD}}_{\mathrm{Del}}$ suggest a heterogeneous system.

Figure 2 shows the classification diagram indicating the type of dispersion found for any given pair of values for $\overline{\mathrm{AD}}_{\text {Del }}$ and area fraction, $A_{f}$. The dashed line shows the crossover between good and poor dispersion, in which materials falling beneath this line are classified with good dispersion and conversely those materials falling above are classified with poor dispersion.

Experimentally it is very difficult to take enough micrographs of a material to obtain a precise estimate of the $\overline{\mathrm{AD}}_{\text {Del }}$ due to the large number of micrographs required (in [16] it was suggest that it could require as many as 100 micrographs; however, this requirement should be

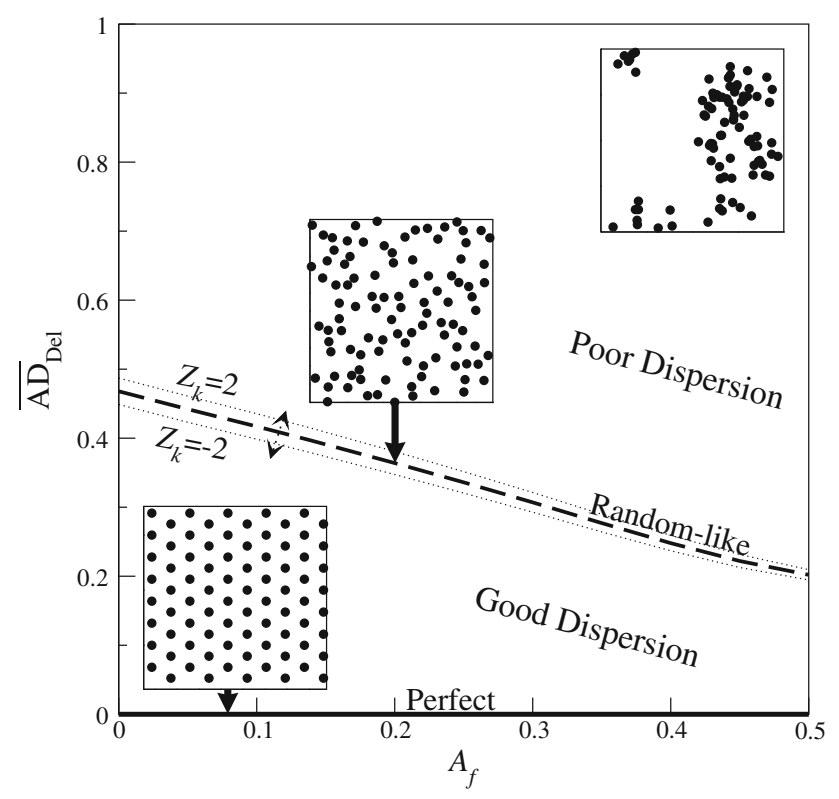

Fig. 2 Classification diagram of dispersion for given combinations of Area Disorder and area fraction. The insets show sample micrographs for a (bottom-top) lattice-like, random or clustered system expected to decrease with lower magnification micrographs which pick up more particles and cover larger areas). In any single micrograph, the measured value for the Area Disorder will vary around the mean value due to natural fluctuations in local area fraction. Hence, it is unlikely that any two independently placed micrographs of the same material will give identical values for $\mathrm{AD}_{\mathrm{Del}}$. Furthermore, it is possible to obtain a value of $\mathrm{AD}_{\text {Del }}$ greater than the true mean value. Thus, it is important to expect some intrinsic imprecision in the measured value of $\mathrm{AD}_{\text {Del }}$ from a single micrograph due to sample variation and account for this in the analysis. This continues to hold true to a significant, but lesser, extent when estimating the $\overline{\mathrm{AD}}_{\mathrm{Del}}$ from a limited number of independently placed micrographs.

To correctly identify the dispersion of the material a hypothesis test is performed to determine the likelihood that the set of micrographs represent a particular type of dispersion.

\section{Hypothesis test}

The hypothesis test is a simple two-sided $z$-test where the estimated mean value of $\mathrm{AD}_{\text {Del }}$ measured from $k$ sample micrographs, e.g. $\overline{\mathrm{AD}}_{k}=\sum_{i=1}^{k} \mathrm{AD}_{i} / k$ (here $\mathrm{AD}_{i}$ is the measured Area Disorder for the $i$ th micrograph), is examined against the random variation of the null hypothesis. An alternative estimate for the mean can also be obtained through the simultaneous analysis of all Delaunay triangles from the collection of $k$ micrographs, as is discussed in Sect. 'An alternative ensemble estimate for $\mathrm{AD}_{\mathrm{Del}}$ ', and the same hypothesis test used.

The null hypothesis states that the nanoparticles (with the material's dimensions being very much larger than the micrograph) are homogeneously randomly distributed across the material with a calculable mean $\left(\mu_{\mathrm{R}}\right)$ and standard deviation $\left(\sigma_{\mathrm{R}}\right)$ for the Area Disorder of a sample region. If the material obeys the null hypothesis then, assuming that the micrographs are chosen independently, in $95 \%$ of cases $\overline{\mathrm{AD}}_{k}$ will have a value that lies within two standard errors (defined as $\sigma_{\mathrm{R}} / \sqrt{k}$ ) of the expected mean $\mu_{\mathrm{R}}$ under the null hypothesis. This is based upon the bell-shaped error curve of random variation expected for $\mathrm{AD}_{\text {Del }}$ in the null hypothesis.

The test statistic $Z_{k}$ is defined as

$Z_{k}=\sqrt{k}\left(\overline{\mathrm{AD}}_{k}-\mu_{\mathrm{R}}\left(A_{\mathrm{f}}\right)\right) / \sigma_{\mathrm{R}}\left(A_{\mathrm{f}}, N\right)$.

The null hypothesis is rejected at the $5 \%$ significance level when $\left|Z_{k}\right|>2$. In such cases either the material is likely to be well dispersed, such that $Z_{k}<-2$ or alternatively the material is likely to be poorly dispersed, such that $Z_{k}>2$. When $\left|Z_{k}\right|<2$, the system is indistinguishable from 
randomly dispersed. The dotted lines in Fig. 2 show an example of the tolerance boundaries, within which the measurement is indeterminate from random. As either $N$ or the number of micrographs sampled over increases, then these boundaries contract towards the dashed line. That said the variation in value of $\mathrm{AD}_{\mathrm{Del}}$ for random dispersion is small compared to the overall range in values for $\mathrm{AD}_{\mathrm{Del}}$.

Values for $\mu_{\mathrm{R}}$ and $\sigma_{\mathrm{R}}$ are determined using theoretical models. The most realistic approach is to treat particles as having hard non-overlapping cores, which will be called the random hard-core model (RHM). Here, particles are placed one at a time randomly without bias anywhere in the system subject to the constraint that a particle must not overlap with any other. In these models, the values of $\mu_{\mathrm{R}}$ and $\sigma_{\mathrm{R}}$ are dependent on the area fraction $A_{f}$ and weakly dependent on the number of particles present.

In the absence of known analytical solutions, computer simulations can be performed to numerically calculate the value of $\mathrm{AD}_{\mathrm{Del}}$ for different values of $A_{f}$. When $N>100$, then it is found that $\mu_{\mathrm{R}}\left(A_{f}\right)$ is approximately independent of $N$. An analytical solution can be derived at the limit of point-like particles where $A_{f}=0$. In this case $\mu_{\mathrm{R}}(0)=0.468$ and $\sigma_{\mathrm{R}}(0, N)=0.214 N^{-0.5}$. The set of mean values with respect to area fraction forms the dashed crossover boundary seen in Fig. 2. The boundary is not linear with respect to $A_{f}$ but can be well approximated with the linear relation:

$$
\begin{aligned}
\mu_{\mathrm{R}}\left(A_{\mathrm{f}}\right) & \approx \mu_{\mathrm{R}}(0)-2 A_{f}\left(\mu_{\mathrm{R}}(0)-\mu_{\mathrm{R}}(0.5)\right) \\
& \approx 0.468-0.532 A_{f} .
\end{aligned}
$$

In general $\sigma_{\mathrm{R}}\left(A_{f}, N\right)$ can be factorised as a product of variables of the form $\sigma_{\mathrm{R}}\left(A_{f}, N\right) \simeq S_{0}\left(A_{f}\right) N^{-1 / 2}$ with $S_{0}\left(A_{f}\right)$ found to be well fitted by the simple function

$S_{0}\left(A_{f}\right) \approx 0.214-0.427 A_{\mathrm{f}}^{1.71}$,

as illustrated by the dashed line in Fig. 3.

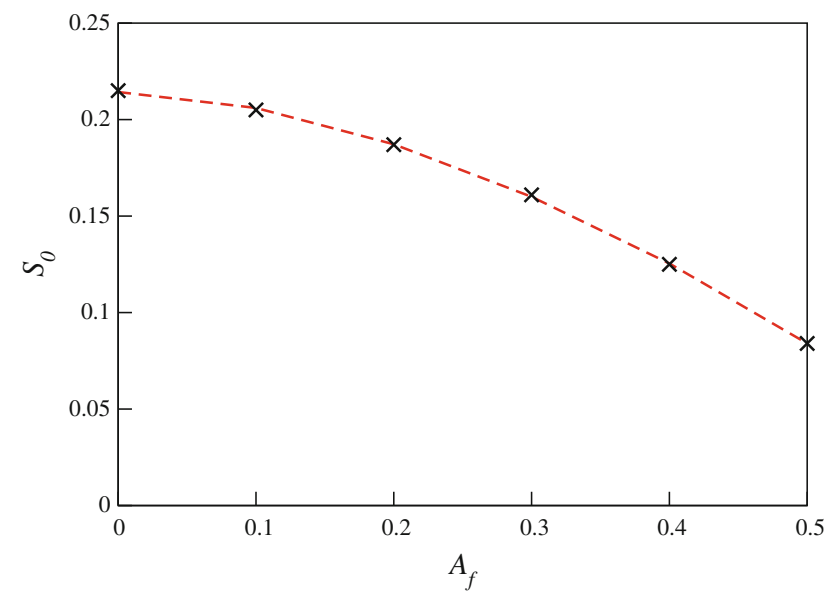

Fig. 3 Trend of $S_{0}$ for null hypothesis. The dashed line is the prediction function prescribed in the main text
With $A_{f}$ known, for example, when predefined by the global properties of the material, and all particles are identifiable through image analysis, then using these values for $\mu_{\mathrm{R}}$ and $\sigma_{\mathrm{R}}$ allows the technique of measuring $\mathrm{AD}_{\mathrm{Del}}$ and calculating $Z_{k}$ to be used as stated. That said, care must be taken when applying the technique to samples which do not have all the particles identified, as will be shown later.

\section{Experimental method}

\section{Materials}

In this article, a particulate-modified epoxy polymer is used [23]. This is produced by mixing an epoxy resin, a curing agent and various particles. The epoxy resin used was 'LY556' supplied by Huntsman, UK, which is a standard diglycidyl ether of bis-phenol A (DGEBA) with an epoxide equivalent weight (EEW) of $185 \mathrm{~g} / \mathrm{eq}$. The curing agent was an accelerated methylhexahydrophthalic acid anhydride, 'Albidur HE 600' (anhydride equivalent weight of 170 g/eq), supplied by Nanoresins, Geesthacht, Germany. This mixture is poured into release-coated moulds to produce plates, and cured at $90^{\circ} \mathrm{C}$ for $1 \mathrm{~h}$ then post-cured at $160^{\circ} \mathrm{C}$ for $2 \mathrm{~h}$. In the resulting microstructure of the crosslinked polymer, the epoxy forms the matrix.

Two particle compositions have been used, the first containing silica nanoparticles covering an area fraction $A_{f}=0.137$, and the second containing both silica nanoparticles at $A_{f}=0.066$ and rubber microparticles with an area fraction of 0.106. Nanoresins supplied silica nanoparticles at a concentration of $40 \mathrm{wt} \%$ in a DGEBA epoxy resin $(\mathrm{EEW}=295 \mathrm{~g} / \mathrm{eq})$ as 'Nanopox F400'. The mean particle radius of the silica is given by the manufacturer as $\bar{r}=10 \mathrm{~nm}$. Nanoresins also provided the carboxyl-terminated butadiene-acrylonitrile (CTBN) epoxy adduct with a rubber concentration of $40 \mathrm{wt} \%$ in a DGEBA epoxy resin, as 'Albipox 1000' (EEW = $330 \mathrm{~g} / \mathrm{eq}$ ), which forms the rubber microparticles upon curing. Further details of these materials and their preparation are given by Hsieh et al. [24].

\section{Micrographs}

The test material is prepared by mounting it in a RMC Products ultramicrotome and slicing using a diamond knife along the longest plane to create a smooth surface. This surface is scanned using a MultiMode scanning probe atomic force microscope (AFM) from Veeco equipped with a NanoScope IV controlled J-scanner and a $5 \mathrm{~nm}$ silicon probe in tapping mode. The AFM records a pair of images depicting the phase (hardness) and height of the scanned area. The phase image is often the clearest and hence most 
suitable image for analysis, with the epoxy making up the predominant background tone. The hard silica nanoparticles provide distinct lighter tone responses whilst the soft rubber particles are darker in tone. Lower level particles, i.e. those just below the surface, add additional noise to the background by generating local high intensity maxima. Overall the intensity of the nanoparticles in the phase image varies depending on their height with respect to the surface.

\section{Image processing}

From a micrograph, the particles are identified using an automated computer routine which broadly consists of three stages. First, the image is processed converting: the micrograph into greyscale; suppressing the background noise by smoothing the image with a median filter to reduce small fluctuations of intensity; and adjusting the contrast level of the micrograph to maximise the difference between nanoparticles and the background material. The filter's square aperture size is chosen to be $\sqrt{2}$ times the observed particle diameter $\left(\bar{r}_{\text {obs }}\right)$ so that applying the filter to the image will not remove particles. $\bar{r}_{\text {obs }}$ is chosen over the actual particle radius $\bar{r}$ because: (a) particles intercept the sample surface at different heights; (b) the broad probe tip induces particles to appear inflated in size (through convolution with probe tip [25] the form of which depends on the exact probe shape and height of particle above the surface). Hence, the mean particle radius is assumed (for simplicity) to be read as

$\bar{r}_{\text {obs }}=\sqrt{2 / 3} \bar{r}+d_{\text {prob }}$,

with $d_{\text {prob }}=5 \mathrm{~nm}$ being the width of the probe (a worst case scenario which ignores the complex relationship between the profiles of the probe and particle) to give a ratio $\bar{r}_{\text {obs }} / \bar{r}=1.32$.

Second, particles are identified through the operator's chosen segregation method, as described below. Third, the particles found are corrected by splitting overly large particles into components and removing likely false positives. The inclusion of false particles, generated through the computer algorithm, is reduced by pruning those particles that lie closer than the mean particle diameter $\left(2 \bar{r}_{\text {obs }}\right)$ to another point. Additional false positives are caused by any cut lines present on the material's surface after cutting with the diamond knife and scan aberration of the AFM. Where scanning aberrations are present (indicated in the micrographs by vertical banding along the righthand side), as is seen in many of the micrographs of the silica-rubber modified composite, the effects are limited by removing potential particles which have a minor axis length (before splitting) of less than two pixels wide.
The chosen segregation process automates particle selection to avoid manual picking - a laborious task which is impractical for sampling large numbers of micrographs. This will inevitably lead to some errors in particle identification through the addition (through false positives and fragmentation) or exclusion of some particles. Two methods of automated segregation are used in this article; in both, the particles are assumed to lie at the focal point of local maximum intensities.

In the simple approach (SimAlg), the algorithm finds every local maximum, regardless of strength above the background, and assigns this to be the particle's centre, i.e. each maximum represents a particle. This method is susceptible to large numbers of false positives when fluctuations of intensity in the background of the micrograph are stronger than can be removed using the digital filter.

The second, more selective, computer algorithm (SelAlg) identifies the hull of a particle by finding all connected image pixels that lie around a local maximum which have intensities no more than a set threshold value, $T$, different from the peak value. Alternatively, particles can be found by repeating the same process using a threshold value of $255-T$ (where 255 is the maximum intensity) around a local minimum and taking the complement of the resultant binary image. The threshold value $T$ is adjusted until the number of particles found by each method is approximately equal. Particles are identified either from both methods and combined to form a complete list of particles (in the case of silica nanoparticle modified composite) or just from the local maximum (in the case of silica-rubber particle modified composite). At this stage duplicate particles are not removed. Additional splitting of conjoined particles is performed by assuming that the centre of mass points of a joint set of particles arise at the local maxima within their hull. Larger objects, over twice the maximum expected size estimated by $\bar{a}=\pi\left(\bar{r}_{\mathrm{obs}}\right)^{2}$ for spherical particles, are broken into smaller particles by assuming each constituent particle has a centre of mass that lies at a local intensity maximum within the hull of the object.

Figure 4 shows the output of the steps taken by the selective computer routine to convert a micrograph image to centre of mass points which are then used to generate a Delaunay network.

The image processing and subsequent analysis of batches of micrographs are performed together using a single automated MATLAB program with standard functions. Other existing routines of image analysis can be made using commercially available software (which may require more complex code or separate analysis of each micrograph). For example, greyscale binarization using a threshold value can be used instead of SelAlg, as long as false positives are eliminated and no systematic regional 
(a)

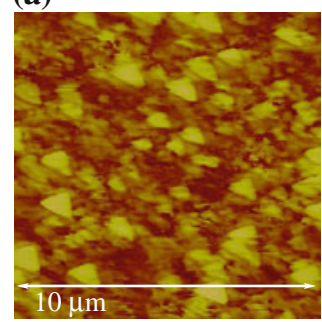

(b)

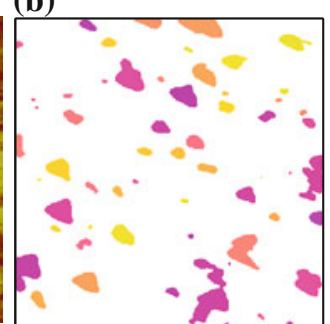

(c)

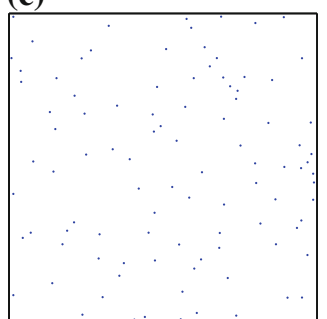

(d)

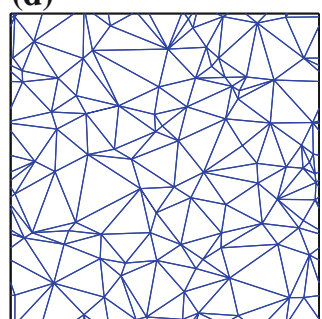

Fig. 4 Stages of image processing (based upon SelAlg): (a) Original micrograph, (b) identified hull of particles, (c) centre of mass points and (d) corresponding Delaunay network

bias is placed on the particles picked out through selection. The difficulty lies in automation of the binarization method as it is uncertain how to optimise the threshold value. Predominantly, this is done by matching the measured area fraction of nanoparticles with the known area fraction of the material. However, this ignores the fact that significant over-sizing of the particle can occur due to finite probe tip size, and that particles lower in depth within the material surface are darker in colour so less likely to be picked out as well as being less well defined.

The purpose here of the chosen routines is not endorsement but rather to highlight the differences in measurement of particle arrangement that will arise because of the varying degrees of success that different approaches have at identifying and separating particles. Recognising this means that techniques need to be developed to address and compensate for any bias.

\section{Results and discussion}

In this section, we discuss how the apparent dispersion of the system is affected by the data quality given in the micrograph and methods for handling the output to maintain an objective perspective. The data quality is affected by both the initial experimental conditions of the microscope when measurement of the material is made and the computation routine of identifying and locating particles from the resultant micrograph.

Strategy for handling incomplete images where not all the particles are visible

When interpreting micrographs it is unlikely that all the particles can be detected without some loss due to the imperfections of the microscope or the limitations in the detection technique used by the segregation algorithm. The data on the particles in these systems can be described as incomplete, i.e. the complete data on all the particles within the micrograph is not known.
In addition, particle incompleteness is caused by the method of preparation used to obtain a suitable smooth surface of the material sample for taking micrograph images. On average, half of the particles present are lost (as the silica nanoparticles are indivisible by the knife), that would have been found if looking along the equivalent plane through the material, onto the opposing face when slicing open the material to reveal a 'flat' surface. Thus, the effect of incompleteness of nanoparticles has very real implications on the result found, even if subsequently perfect conditions exist when taking measurements. So it is important to ask what effect does the loss of particles have on the average measured dispersion quality. Specifically, how robust is the mean value of $\mathrm{AD}_{\mathrm{Del}}$ to small changes in particle numbers, which could be the result of close particles merging to register as one or false positive particle detection? Second, how much change is observed when a large proportion of the particles are missing from the analysis and can it be compensated for?

\section{Test systems}

A random hard-core model (RHM) is used to investigate these two questions. Here, it is the average behaviour over a large number of samples that is studied, not individual samples as the discrepancies between samples and the theoretical expectation can occur through sample variation alone. Let $A_{f}$ be the real average area fraction of material. The expected average number of particles found along a test plane of area $L^{2}$, assuming no particle loss and identical spherical particles with mean radii $\bar{r}$, is calculated from the material's known area fraction by $\bar{N}_{\exp }=$ $3 A_{f} L^{2} / 2 \pi \bar{r}^{2}$. This expression accounts for the particles intercepting the test plane at different heights giving a reduced-size mean cross-sectional area.

Let $A_{f}^{\prime}$ be the observed area fraction from the micrograph where only a fraction $f$ of particles are visible such that $A_{f}^{\prime}=f A_{f}$. Particles are chosen to be invisible through random assignment. Figure $5 \mathrm{~b}$ shows example spatial plots 
Fig. 5 Particle dispersion can appear very different when a fraction of the particles are hidden. This figure shows particles arranged in a lattice, row (a); at random, row (b); in clusters, row (c), when either all $(f=1)$, half $(f=0.5)$ or a quarter $(f=0.25)$ of particles are visible

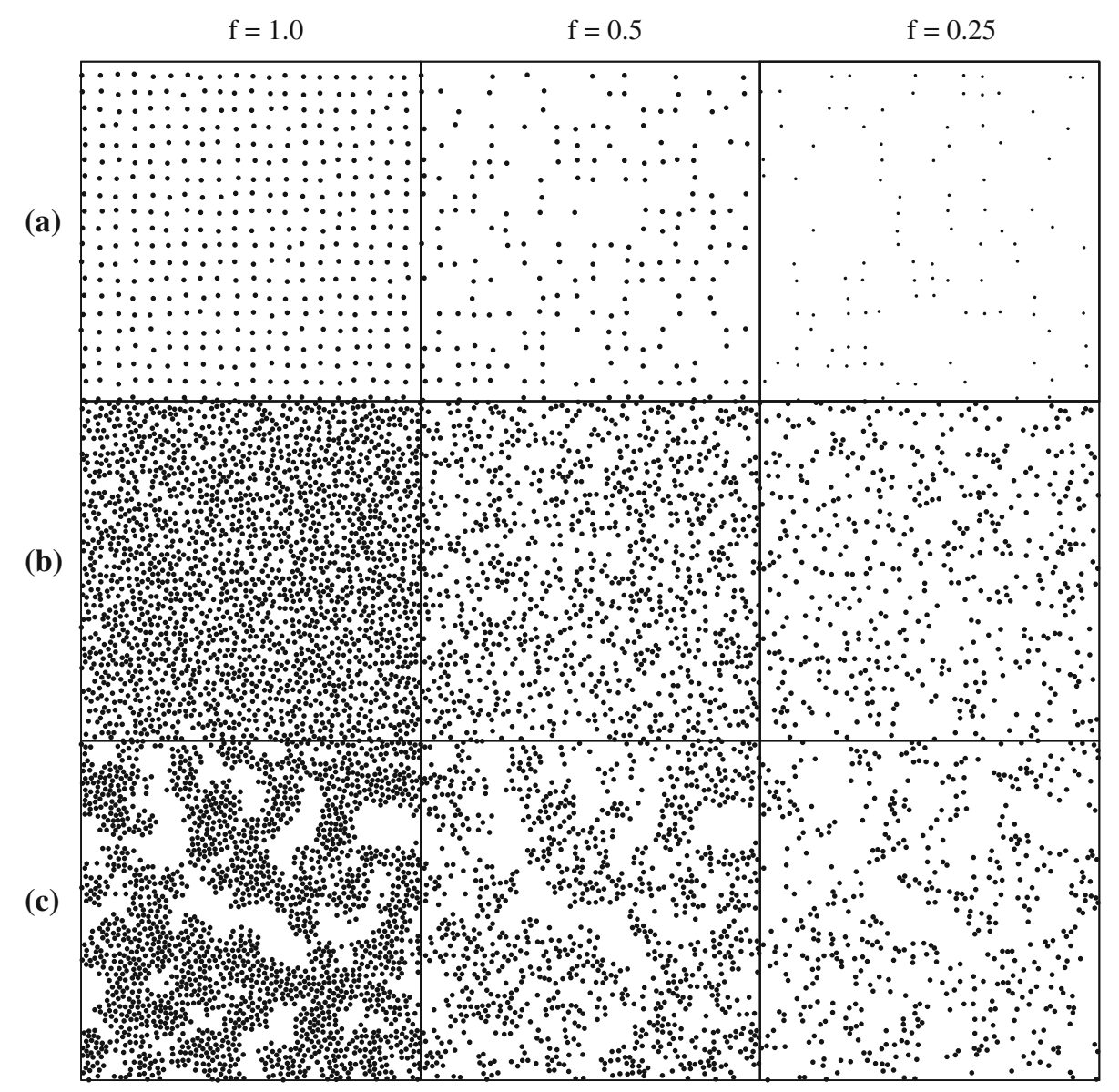

Table 1 Changes in observed value for $\overline{\mathrm{AD}}_{\text {Del }}$ when particles are not visible

\begin{tabular}{llll}
\hline Number of particles & Fraction lost $(\%)$ & $\overline{\mathrm{AD}}_{\text {Del }}$ & $\Delta \overline{\mathrm{AD}}_{\text {Del }}(\%)$ \\
\hline 1000 & 0 & 0.365 & 0 \\
990 & 1 & 0.365 & 0 \\
950 & 5 & 0.373 & 2 \\
900 & 10 & 0.380 & 4 \\
500 & 50 & 0.425 & 16 \\
490 & 51 & 0.426 & 17 \\
450 & 55 & 0.429 & 18 \\
\hline
\end{tabular}

The example is for a RHM with $A_{f}=0.2$

seen when performing this process, and Table 1 tabulates the change in the value of observed mean Area Disorder $\left(\overline{\mathrm{AD}}_{\mathrm{Del}}\right)$ as particles become undetectable using the quantity:

$\Delta \overline{\mathrm{AD}}_{\text {Del }}=\frac{\overline{\mathrm{AD}}_{\text {Del }}(f \equiv 1)-\overline{\mathrm{AD}}_{\text {Del }}(f)}{\overline{\mathrm{AD}}_{\text {Del }}(f \equiv 1)}$.

Here, $\Delta \overline{\mathrm{AD}}_{\mathrm{Del}}$ is referenced against the complete particle case of $f=1$. With small particle loss, up to $10 \%$ of the total number of particles, the value of $\overline{\mathrm{AD}}_{\mathrm{Del}}$ changes by less than $2 \%$. As the expected natural variation of a
RHM is typically of the order of $3 \%$ such small discrepency in $\overline{\mathrm{AD}}_{\text {Del }}$ caused by variation in observed particle numbers is masked. This suggests that the $\overline{\mathrm{AD}}_{\mathrm{Del}}$ measure is sufficiently robust that it will not be thrown off by outliers in the data generated by false-positive particles or a few missing particles. However, with large losses of particles, the value of $\overline{\mathrm{AD}}_{\mathrm{Del}}$ changes significantly compared to that with no loss and requires careful interpretation. A simplistic hypothesis would assume that the RHM remains spatially homogeneous with the remaining particles as would be found for point-like particles (where $A_{f}=0$ ).

Figure 6 shows the equivalent $A_{f}^{\prime}$ versus $\overline{\mathrm{AD}}_{\text {Del }}$ diagram seen in Fig. 2. The dashed line shows the expected division at random dispersion if the measured $\overline{\mathrm{AD}}_{\text {Del }}$ obeyed RHM models with area fractions equal to $A_{f}^{\prime}$ (and no indistinguishable particles). The solid line is the measured value for $\mathrm{AD}_{\text {Del }}$ when $50 \%$ of the particles are undetectable, such that $A_{f}^{\prime}=A_{f} / 2$. The circles shows an example path for $A_{f}=0.4$ traced out as more particles become undetectable, hence lowering the observed packing fraction and changing the recorded $\overline{\mathrm{AD}}_{\mathrm{Del}}$. The arrows point to the direction of increasing particle loss. 


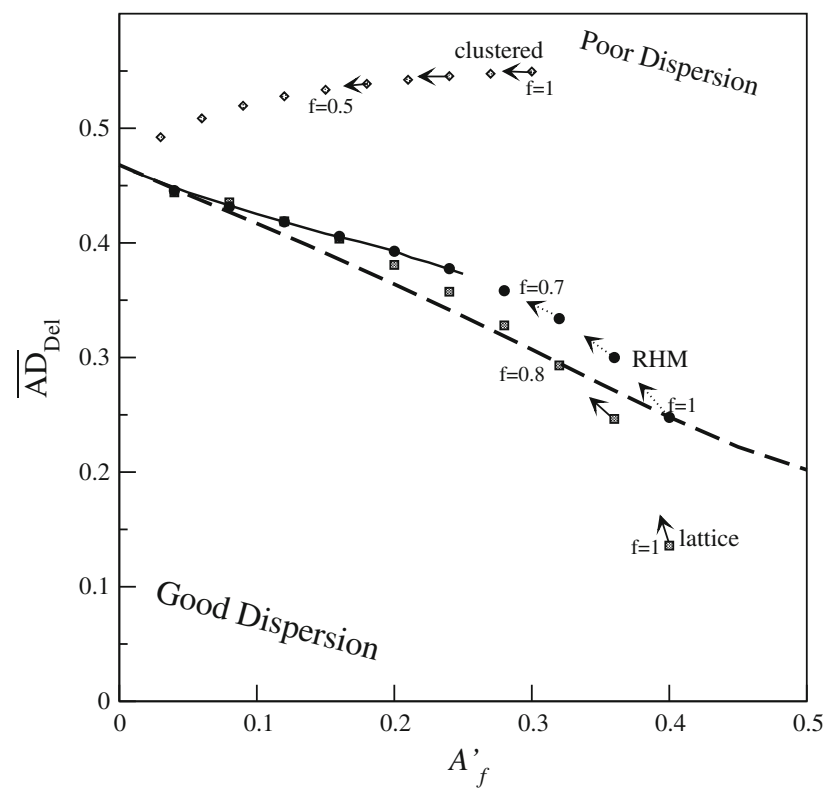

Fig. $6 A_{f}^{\prime}$ versus $\mathrm{AD}_{\mathrm{Del}}$ diagram for three differently dispersed systems. The dashed line gives the anticipated behaviour for the RHM if the hidden particles have no influence on the positions of the remainder. The solid line is the actual behaviour for a RHM when $50 \%$ of the particles are lost

These results demonstrate that with the absence of select particles from a spatially random distribution of particles then the arrangement of the remaining particles registers as more clustered than expected for a RHM with the effective area fraction equal to $A_{f}^{\prime}$. It shows that although some of the particles might be undetectable in a micrograph their effect on the detectable particles can be important. It is worth bearing this in mind as it means that if the measured $\overline{\mathrm{AD}}_{\text {Del }}$ is less than that from a RHM using $A_{f}^{\prime}$ then it will also be less than that found from a RHM using $A_{f}$ with the equivalent fraction of particles lost. In general, the converse cannot be said: if the spatial arrangement of particles fails to register as good dispersion then it does not necessarily follow that it is poorly dispersed.

However, when $A_{f}$ is small, then the measured value of $\overline{\mathrm{AD}}_{\text {Del }}$ (for a RHM using $A_{f}$ and with $f<0.5$ particles visible) is effectively equivalent to the RHM with area fraction equal to $A_{f}^{\prime}$ and all particles observable (as can be seen in Fig. 6 by the convergence of dashed and solid lines when $\left.A_{f}^{\prime}<0.1\right)$. This is beneficial as typically the area fraction of the composite materials under investigation is less than 0.2 , which corresponds to $A_{f}^{\prime}<0.1$, and the observed $f$ is much smaller than the 0.5 upper limit for AFM images. Hence, for these systems it is sufficient to substitute a RHM using $A_{f}^{\prime}$ for the more correct RHM using $A_{f}$ with $(1-f)$ particles undetectable.

This increase in observed value for $\overline{\mathrm{AD}}_{\text {Del }}$ is found for other well-dispersed particle arrangements. The most extreme case is for the perfect lattice. For example, take a near square-lattice array of particles-see the spatial plots in Fig. 5a-with particles having small random perturbations away from the lattice location such that the minimum possible distance between particles is equal to the diameter of the particles for $A_{f}=0.4$. The squares in Fig. 6 show the various observed measurement for $\overline{\mathrm{AD}}_{\text {Del }}$ as $f$ is varied. The value of $\overline{\mathrm{AD}}_{\text {Del }}$ increases rapidly from near 0 as $A_{f}^{\prime}$ decreases. However, it is always bounded by the equivalent behaviour of RHM, in this case that of $A_{f}=0.4$ again shown by the circular points.

With clustered systems, the value of $\overline{\mathrm{AD}}_{\text {Del }}$ decreases but remains greater than that expected for point-like randomly distributed particles. An example of this is shown by the diamonds in Fig. 6 and the spatial plots in Fig. 5c in which 2,000 particles, in clusters of 10 with each particle Gaussian-distributed about a randomly positioned centre but limited by the constraints of particle size.

\section{Modified z-tests}

Consequently for these materials, a $z$-test can still be meaningfully performed on the micrograph using $A_{f}^{\prime}$ as the area fraction and $\bar{N}_{\text {found }}$ for the number of particles. The test statistic is denoted as $Z_{k}$ (low) for clarity. The exact circumstances for which particles lose visibility are dependent on a complex combination of the method of sample preparation, the set-up of the microscope, the position of the material under observation and the analysis program used. Hence, it is difficult to evaluate $f$ before taking the micrographs and instead $f$ is estimated afterwards from the set of $k$ micrographs using

$f \simeq \frac{\bar{N}_{\text {found }}}{\bar{N}_{\text {expand }}}=\left(\frac{2 \pi \bar{r}^{2}}{3 L^{2} A_{f}}\right) \bar{N}_{\text {found }}$,

where $\bar{r}$ is the mean radius of a particle given by the manufacturer and $\bar{N}_{\text {exp }}$ is the expected number of particles. From $f$, the effective area fraction can be calculated:

$A_{f}^{\prime} \simeq f A_{f}=\left(\frac{2 \pi \bar{r}^{2}}{3 L^{2}}\right) \bar{N}_{\text {found }}$.

Calculation of $A_{f}^{\prime}$ cannot be done through direct measurement of particle size because of the inaccuracies in measured size brought on by the use of the finite-tipped probe and the ill-defined particle boundary given by the detection algorithm. When $Z_{k}$ (low) $>-2$, a second $z$-test can be performed equating $A_{f}^{\prime}=0$, with the test statistics denoted as $Z_{k}$ (upp), to see if the system is likely to be dispersed poorly.

The implied consequence of the analysis shown here should give pause for thought. Given that the measured value of $\mathrm{AD}_{\mathrm{Del}}$ (and by extension the observed dispersion) 
is sensitive to the fraction of particles that are visible, then the absolute value of $\mathrm{AD}_{\mathrm{Del}}$ is of less use to the investigator than its relative position compared with the values of other test materials (which informs us about any correlations in behaviour). This underlines the importance of measuring, as near as possible, each sample using an identical method (with respect to sample preparation, microscope used and computer algorithm used for analysis) to ensure a fair comparison between results.

Selecting the necessary magnification level of the microscope to achieve a reliable estimate of $\mathrm{AD}_{\mathrm{Del}}$

The effects of the microscope's magnification on the measurement of dispersion is complex. Varying the resolution of the micrograph will change both the extent of nanoparticle microstructure seen and the ability of the image analysis to distinguish between nanoparticles. Specifically, for a given sample of material, those micrographs taken by the microscope at lower magnifications will show larger-scale spatial features, which ideally leads to stronger indications of lattice-like or heterogeneous dispersions, but also are more strongly affected by the arbitrary merging and loss of nanoparticles due to the discrete nature of the pixel resolution.

To experimentally study the effects of the magnification level upon $\mathrm{AD}_{\text {Del }}$, the $\mathrm{AFM}$ magnification level for the two test materials is chosen such that the resultant micrographs show a surface area of the material that spans a length $L$ of either $1,1.5,2.5,3.5,5,7.5$ or $10 \mu \mathrm{m}$. If desired $L$ can be converted to a unit-less measure, $L^{\prime}$, by multiplying by the square-root of the average number density, $\lambda=N / L^{2}=$ $3 A_{f} / 2 \pi \bar{r}^{2}$, expected for a given area fraction and particle size.

\section{Silica nanoparticle modified composite}

In this section, the silica nanoparticle modified composite is analysed using the previously described method and the type of dispersion determined with the aid of the $Z_{k}$ (low) test statistic. The composite is sampled at six different locations (four broadly chosen to be located close to the four corners and two close to the centre of the material's microtomed surface) with an image taken at each of the six magnification levels (given above) to produce 36 micrographs (each micrograph consists of 512 by 512 pixels giving a pixel length of $l=L / 512 \mathrm{~m}$ ). A measurement of $\mathrm{AD}_{\text {Del }}$ is made for each individual micrograph to demonstrate the spread of values for $\mathrm{AD}_{\text {Del }}$ obtainable from a single snapshot, and also an estimate for the average behaviour at each magnification scale is gathered.
Examples of the micrographs obtained for various magnifications are shown in Fig. 7a-f.

Tables 2 and 3 tabulate the analysis data obtained using the selected and simple computer algorithm from the set of micrographs with various magnification levels. Notice that the fraction of particles visible in either case is much less than the theoretical maximum of a half (expected for AFM images) when compared to $N_{\exp }$ (equivalent to $1-f$ ) and implies that not all the particles can be identified in the micrograph (the exact fraction of particles measured will depend on the approach taken during image processing, as is demonstrated here with SimAlg picking out between two and three times as many particles as SelAlg).

The standard error of the $\mathrm{AD}_{\mathrm{Del}}$ values $\left(\mathrm{SE}_{\mathrm{AD}}\right)$ gauge the variations in measurement of individual micrographs away from the estimated mean value. A relatively large change in $\mathrm{SE}_{\mathrm{AD}}$ is seen between $L=1$ and $L=2.5 \mu \mathrm{m}$, whilst lesser improvement is obtained by further reductions of magnification. An exception occurs with $L=10 \mu \mathrm{m}$ where the variation in measurement worsens. This is likely to be due to inaccuracies in particle position brought on by the smallness of particle size (diameter of $20 \mathrm{~nm}$ ) when compared to the pixel width of the micrograph $(19.5 \mathrm{~nm})$. On a note of caution, when $\bar{N}<100$, then the simplification of $\mu_{\mathrm{R}}$ that goes into $Z_{k}$ is invalid.

Figure 8 plots the results for the observed number of particles, Area Disorder and $Z_{k}$ (low) versus the image length $L$. The measured data from individual micrographs are shown as cross (for SelAlg) and plus points (for SimAlg) whereas the means are shown as joined circle and square points, respectively. The observed average number of particles increases approximately linearly with $L^{2}$, see Fig. 8a, but is much less than the theoretical maximum for the AFM micrograph shown by the dashed line as was already alluded to from the tables and is clearly less than that expected from using the material's known area fraction shown by the dotted line.

Figure $8 \mathrm{~b}$ shows the trend in the observed value of $\mathrm{AD}_{\text {Del }}$ for the different magnification levels. The dotted line in Fig. 8b again indicates the danger of naively using the material's known global area fraction without compensating for the incompleteness of visible particles present in the micrographs. By doing so we strikingly reduce the difference between the observed responses of $A D_{\text {Del }}$ in the test material and that for the corresponding RHM. Most importantly depending on the choice of segregation algorithm, the system can be inaccurately read as either poorly dispersed (as would be read from SelAlg) or well dispersed (as would be read from SimAlg) and hence the analysis would be sensitive to the computer program used.

Using the correct adjustment for the RHM, shown in Fig. $8 \mathrm{~b}$ by the dashed and point lines (with symbols 
Fig. 7 (a, d) Show micrographs at $L=1 \mu \mathrm{m}$. Similarly (b, e) illustrate micrographs at a different location at $L=2.5 \mu \mathrm{m}$ and $(\mathbf{c}, \mathbf{f})$ micrographs at $L=5 \mu \mathrm{m}$. The dots in the micrographs give the determined centre of mass points for the recognised nanoparticles determined using SelAlg $(\mathbf{a}-\mathbf{c})$ and SimAlg $(\mathbf{d}-\mathbf{f})$
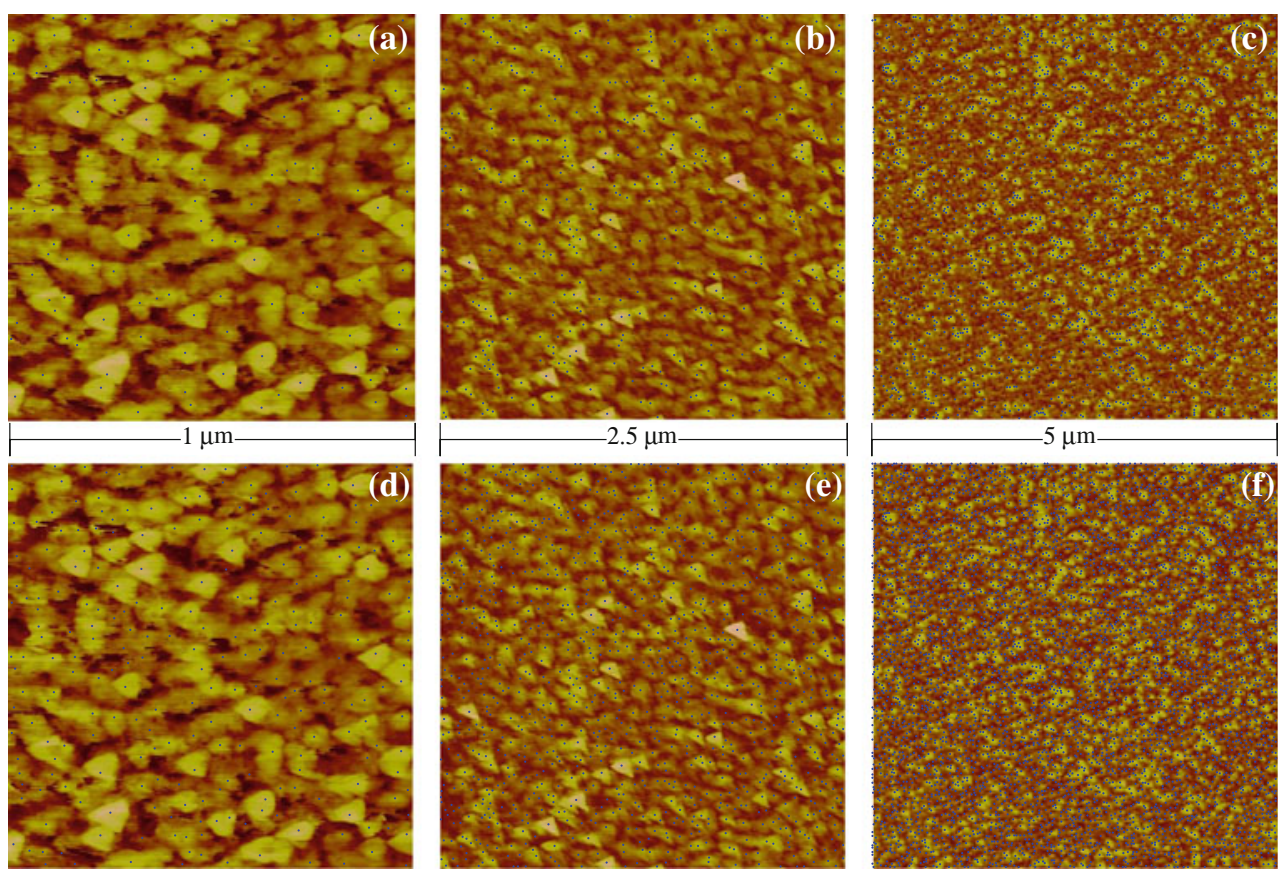

Table 2 Measured statistics for silica nanoparticle modified composite using SelAlg

\begin{tabular}{llllll}
\hline$L(\mu \mathrm{m})$ & $\bar{N}$ & $\bar{f}$ & $\overline{\mathrm{AD}}_{k}$ & $\mathrm{SE}_{\mathrm{AD}}$ & $Z_{k}(\mathrm{low})$ \\
\hline 1.0 & 101 & 0.154 & 0.4023 & 0.0097 & -6.2788 \\
2.5 & 496 & 0.121 & 0.4159 & 0.0067 & -11.0523 \\
3.5 & 929 & 0.116 & 0.4133 & 0.0057 & -16.1673 \\
5.0 & 2153 & 0.132 & 0.4265 & 0.0042 & -16.9616 \\
7.5 & 3924 & 0.107 & 0.4147 & 0.0028 & -32.6654 \\
10.0 & 8007 & 0.122 & 0.4266 & 0.0064 & -33.3362 \\
\hline
\end{tabular}

Table 3 Statistics measured using SimAlg

\begin{tabular}{llllll}
\hline$L(\mu \mathrm{m})$ & $\bar{N}$ & $\bar{f}$ & $\overline{\mathrm{AD}}_{k}$ & $\mathrm{SE}_{\mathrm{AD}}$ & $Z_{k}$ (low $)$ \\
\hline 1.0 & 259 & 0.396 & 0.3652 & 0.0030 & -13.8140 \\
2.5 & 1468 & 0.359 & 0.3640 & 0.0060 & -34.5558 \\
3.5 & 2662 & 0.332 & 0.3640 & 0.0052 & -47.6969 \\
5.0 & 5644 & 0.345 & 0.3619 & 0.0062 & -70.4391 \\
7.5 & 8526 & 0.232 & 0.3394 & 0.0070 & -118.7651 \\
10.0 & 17315 & 0.265 & 0.3466 & 0.0080 & -155.0225 \\
\hline
\end{tabular}

corresponding to that used for means), a much stronger case can be made that the nanoparticles in the test material are better-dispersed-than-random. This is shown most clearly with the values of $Z_{k}$ (low). Although overall $\mathrm{AD}_{\text {Del }}$ varies with $L$ the likelihood that it resulted by chance from a RHM decreases, as shown by the increasingly negative values for $Z_{k}$ (low) in Fig. 8c. This indicates that the evidence that the material is better-dispersed-than-random is strengthening with $L$. With a $Z_{k}$ (low $)=-2$ then only $2.5 \%$ of samples of a equivalent RHM will have $\mathrm{AD}_{\text {Del }}$ with a lower value than has been measured. By reducing $Z_{k}$ (low) to -5 , this drops to $0.00001 \%$ of RHM samples. Hence, a response of $Z_{k}$ (low) over -10 makes it extremely unlikely that the observed properties represent a set of randomly dispersed nanoparticles and thus must be more regularly dispersed.

Thus, it can be concluded from the results that particularly promising magnification levels lie approximately between 2.5 and $5 \mu \mathrm{m}$. For these resolutions, the standard error of $\mathrm{AD}_{\text {Del }}$ between samples is kept at an acceptable level whereas the fraction of particles visible in the micrographs remains relatively constant. Furthermore, the mean value of $\mathrm{AD}_{\text {Del }}$ also remains reasonably constant to within \pm 0.005 .

\section{Silica-rubber particle modified composite}

In this section, the silica-rubber particle modified composite is analysed using the previously described method involving SelAlg (SimAlg is not considered as it indiscriminately picks out features in both the silica nanoparticles and the rubber microparticles) and the type of dispersion determined with the aid of the $Z_{k}$ (upp) test statistic. Micrographs are taken at six different locations along the material's cut surface, with images captured for each magnification level (each micrograph consists of 382 by 382 pixels giving a pixel length of $l=L / 382 \mathrm{~m}$ ). 


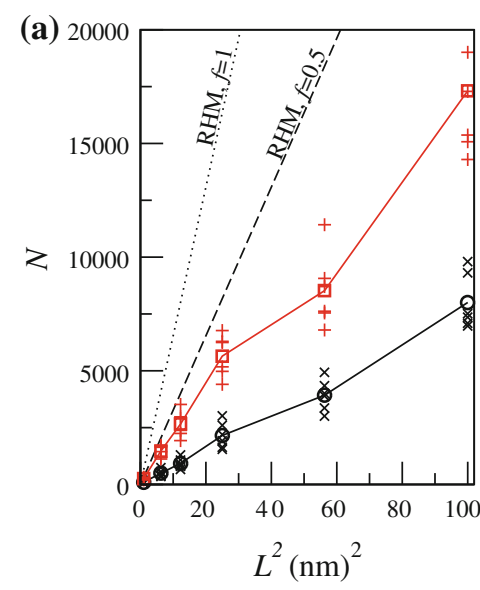

Fig. 8 Measurements for the silica nanoparticle modified composite: (a) the number of particles, (b) the Area Disorder and (c) the $z$-test. The crosses (black) or pluses (red) give observations from individual micrographs for SelAlg and SimAlg, respectively. Similarly, the
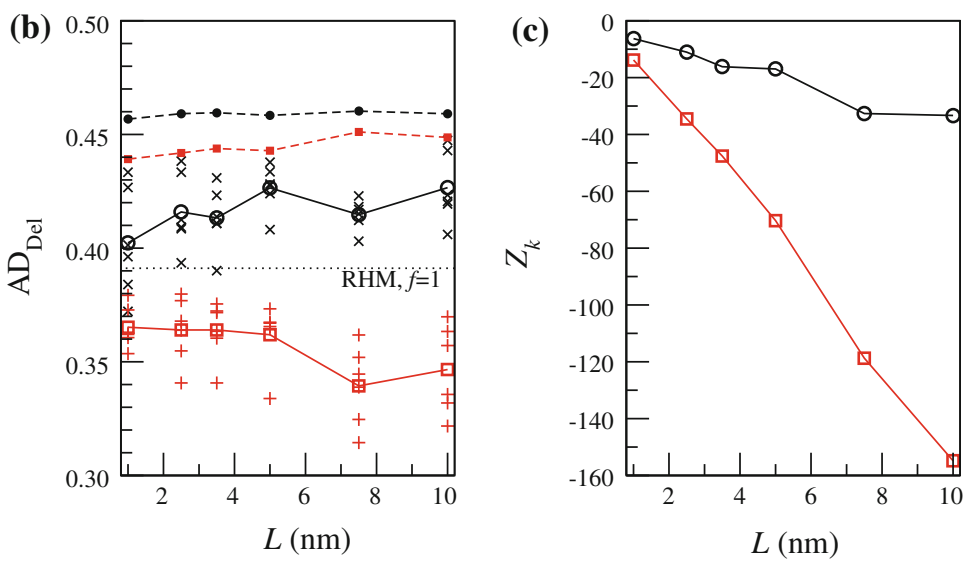

circles or squares connected by solid lines are their means and the same points connected by dashed lines the corresponding corrected RHM (Color figure online)
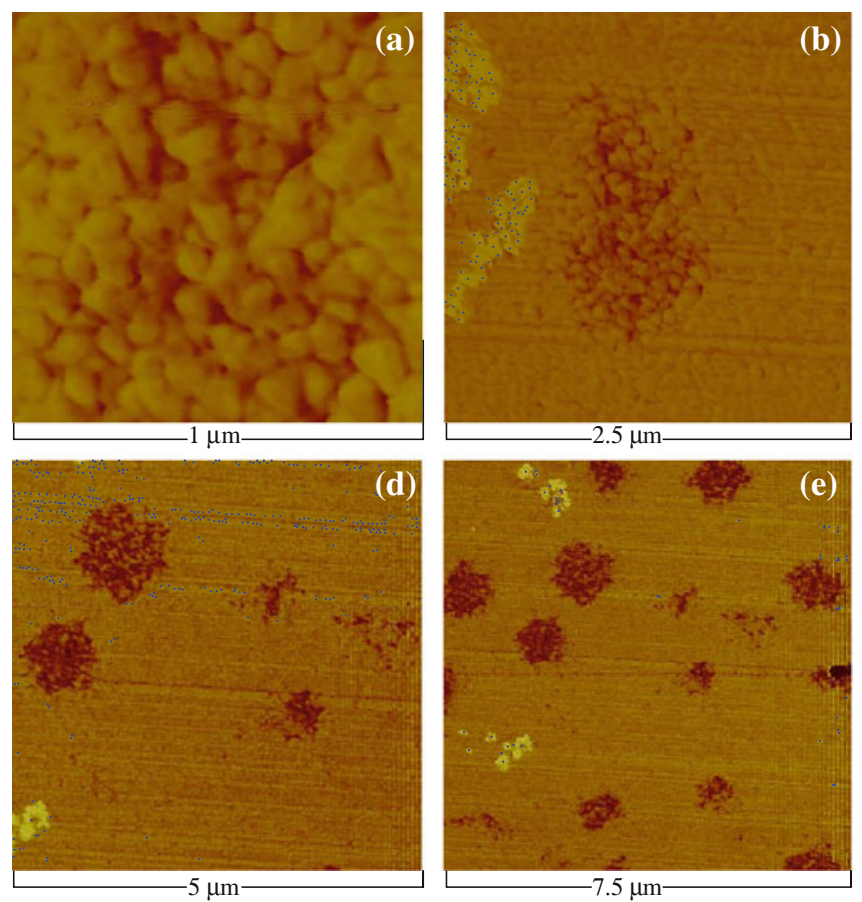
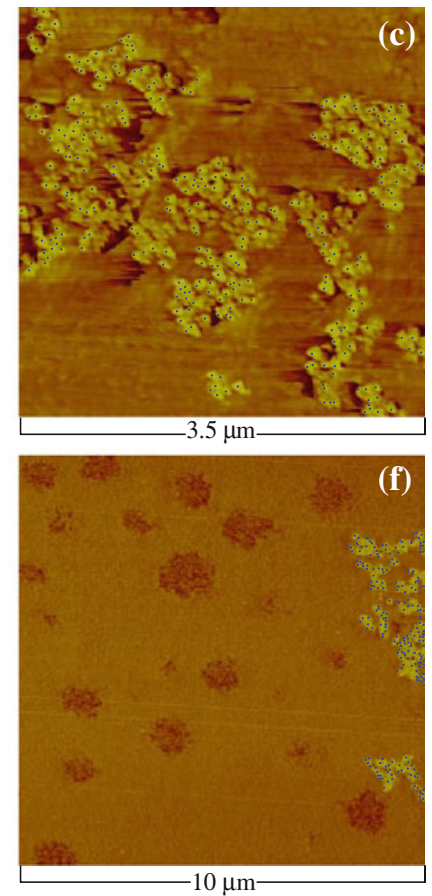

Fig. 9 Micrographs of the silica-rubber particle modified composite with $L=1.0 \mu \mathrm{m}(\mathbf{a}) ; 2.5 \mu \mathrm{m}(\mathbf{b}) ; 3.5 \mu \mathrm{m}(\mathbf{c}) ; 5.0 \mu \mathrm{m}(\mathbf{d}) ; 7.5 \mu \mathrm{m}(\mathbf{e})$ and $10.0 \mu \mathrm{m}(\mathbf{f})$

The micrographs are inspected to decide whether silica nanoparticles are present. Those images with more than three particles are put forward for the computer analysis, whereas those micrographs with insufficient particles have $\mathrm{AD}$ assigned to be 0 . Figure 9 shows an example micrograph for each magnification level. The identified particles' centre of mass positions are overlaid where appropriate. These micrographs illustrate some of challenges met when analysing the material. In Fig. 9a, no nanoparticles are present and instead the micrograph has haphazardly been focused on the internal structure of a rubber microparticle. In Fig. 9d, the computer algorithm selects the cutting lines in preference to the nanoparticles. Whilst in Fig. 9e, some of the scanning aberrations are identified as particles (although this has been greatly restricted and in other cases entirely removed by applying the filtering technique described in the method section).

Table 4 tabulates the analysis data. An indication to the number of micrographs found to have fewer than three nanoparticles is given by the third column of Table 4. For 
Table 4 Measured statistics for silica-rubber particle modified composite

\begin{tabular}{llllll}
\hline$L(\mu \mathrm{m})$ & $\bar{N}$ & $N<3 ?$ & $\overline{\mathrm{AD}}_{k}$ & $\mathrm{SE}_{\mathrm{AD}}$ & $Z_{k}(\mathrm{upp})$ \\
\hline 1.0 & 6.5 & $\mathrm{Yes}(5)$ & 0.1118 & 0.1118 & -10.3940 \\
2.5 & 88.2 & $\mathrm{Yes}(3)$ & 0.3310 & 0.1554 & -14.7250 \\
3.5 & 113.7 & $\mathrm{Yes}(3)$ & 0.3576 & 0.1628 & -13.4499 \\
5.0 & 283.8 & $\mathrm{Yes}(2)$ & 0.5535 & 0.1754 & 16.4796 \\
7.5 & 347.7 & $\mathrm{No}(0)$ & 0.7744 & 0.0442 & 65.4106 \\
10.0 & 451.8 & $\mathrm{No}(0)$ & 0.8321 & 0.0259 & 88.5849 \\
\hline
\end{tabular}

The bracketed numbers give the number of micrographs found to have less than 3 nanoparticles

the four highest magnification micrographs, $L \leq 5 \mu \mathrm{m}$, it is possible to place the micrograph on the material such that none of the nanoparticles are seen, either because we are between clusters or because we have focused inside or around a rubber microparticle. The difference in the apparent dispersion quality between micrographs with nanoparticles and those without exaggerates the magnitude of the standard error of $\mathrm{AD}_{\mathrm{Del}}$. This point is best illustrated by the large reduction in value of the standard error as $L$ increases from $L=5$ to $L=7.5 \mu \mathrm{m}$ which corresponds to whether or not some micrographs contain no nanoparticles.

Figure 10 plots the observed number of particles, Area Disorder and $Z_{k}$ (upp) versus the image length $L$. The mean number of particles seen in a micrograph increases with $L$, as illustrated in Fig. 10a. The optical dilation of particles closely packed into clusters means that it is not possible to partition out all the particles, so no attempt is made to compare the number of particles seen on average with that expected for the system. It is instead felt that that the aim should be to obtain sufficient particle locations to characterise the shapes of the clusters to measure the inter-cluster behaviour correctly. Micrographs with particle data that are found to contain a high number of false positives, sufficient to affect the result, are indicated with additional symbols in Fig. 9. For highly magnified micrographs, background material can be indistinguishable from nanoparticles (the results from these micrographs are indicated by square points). At lower magnifications, false positives are introduced in the presence of any deep cut lines (triangular points) and/or scanning aberration (diamond points) on the micrograph.

Figure $10 \mathrm{~b}$ shows the test values of $A D_{\text {Del }}$. The mean value (shown by the connected circles) increases strongly monotonically with $L$, changing by 0.73 (this is a far stronger response than seen for the silica modified composite where $\overline{\mathrm{AD}}_{k}$ varied only by 0.03 ). The dotted line in Fig. 10b indicates the expected behaviour for null hypothesis, that the nanoparticles of the material are distributed randomly $\left(A_{f}=0\right)$. The effect on $\overline{\mathrm{AD}}_{k}$ of the inclusion of the micrographs with large numbers of false positives depends on the source of the error. In the higher magnification micrographs, identified parts of the background material will push the value of $\mathrm{AD}_{\mathrm{Del}}$ up towards being random-like. Whereas in lower magnification micrographs, inclusion of the false positives due to cut marks or scan aberrations will tend to make the system appear more clustered, with a localised band of false particles in one location of the micrograph.

The effect of the shifting value of $\mathrm{AD}_{\mathrm{Del}}$ on the apparent dispersion quality is strikingly shown by $Z_{k}(u p p)$ in Fig. 10c. When $Z_{k}$ (upp) is above the dotted line, the material is determined to be dispersed in a worse than random manner. An inaccurate conclusion would be drawn by the operator (that the micrographs are not poorly dispersed) when using micrographs with $L \leq 3.5 \mu \mathrm{m}$. Only when using $L$ at least

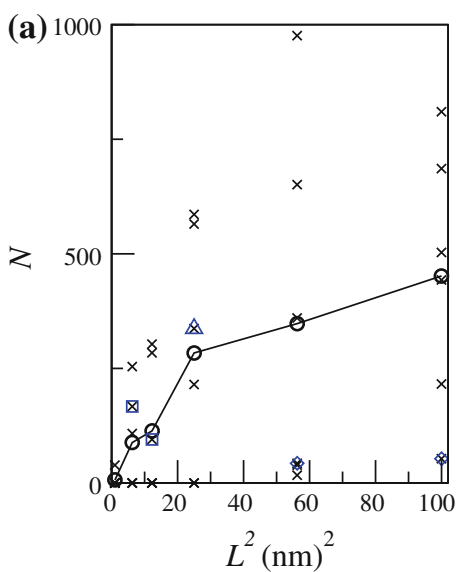

Fig. 10 Measurements for the silica-rubber particle modified composite: (a) the number of particles, (b) the Area Disorder and (c) the $z$-test. The cross points give observations from individual micrographs, and the connected circles give the mean value. Squares,
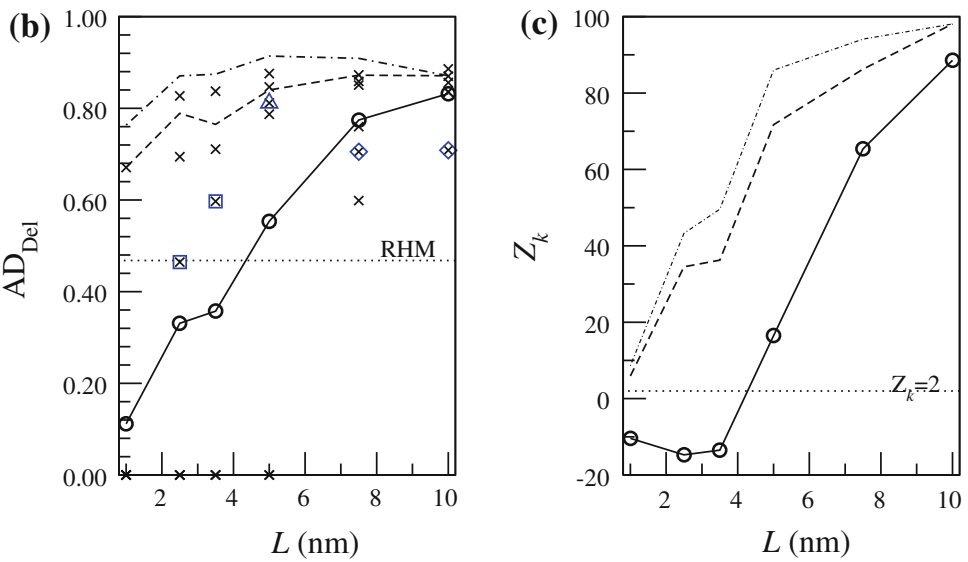

triangles and diamonds are used to indicate suspect micrographs with high numbers of false positives. Dashed lines provide the alternative ensemble approach 
as large as $5 \mu \mathrm{m}$ does the correct dispersion quality become apparent, and we conclude that the material is poorly dispersed. Note here a large negative value for $Z_{k}$ (upp) does not suggest evidence for good dispersion, but merely indicates that the samples show better behaviour than expected for point-like random objects. Only by repeating the hypothesis test using $Z_{k}$ (low) could this be decided upon. In any case, this is unnecessary because the number of particles present in the micrographs for cases where this is true are less than 100. Hence, we would disregard these findings as suspect because the simple arguments used for defining the RHM become invalid for small $N$. The actual value for $\mu_{\mathrm{R}}\left(A_{f}=0\right)$ becomes strongly dependent on $N$ and approaches 0 with decreasing particle number [21].

Thus, it can be concluded that for visually clustered systems the chosen size of the micrograph ought to be selected such that it is much larger than the features of the material, in this case the rubber microparticles, that are of sizes ordering $1 \mu \mathrm{m}$, and the clustered nanoparticles, with the clusters being of a size around $L_{\text {clus }}=2 \mu \mathrm{m}$. The minimum size of the micrograph required to ensure it will contain a cluster can be estimated using:

$L \geq \sqrt{\frac{\pi\left(L_{\text {clus }} / 2\right)^{2}}{A_{\mathrm{f}}}}=\sqrt{\frac{\pi(1 \mu \mathrm{m})^{2}}{0.066}}=6.9 \mu \mathrm{m}$.

Here, we assume that the clusters devoid the surrounding space of nanoparticles (hence we need to select a micrograph large enough that the equivalent RHM would have the same total area of nanoparticles as that which makes up the cluster) and that the clusters are assumed to be circular. We can see that our crude estimate for $L$ matches well with the crossover value that was observed experimentally to be between $L=5$ and $L=7.5 \mu \mathrm{m}$.

\section{An alternative ensemble estimate for $A D_{D e l}$}

We have seen that the use of $\overline{\mathrm{AD}}_{k}$ suffers from two limitations: (1) it is highly influenced by the quality of information available in a micrograph (i.e. including those micrographs with low particle numbers will significantly lower $\overline{\mathrm{AD}}_{k}$ ); (2) large fluctuations in particle numbers between micrographs are not accounted for in $Z_{k}$, where the null hypothesis assumes that every micrograph contains $N$ particles. The use of $\overline{\mathrm{AD}}_{k}$ is necessitated when the micrographs are individually analysed before estimating the average behaviour. However, an alternative estimate can be obtained through analysing all the micrographs simultaneously.

Each micrograph is a small region of the larger material and thus should share the same statistical properties. We record the areas of the Delaunay triangles of each micrograph and collated them into one large table of areas
$\left(\left\{\Omega_{k}\right\}\right)$. From this, the Area Disorder is calculated using the ensemble estimate $\widehat{\mathrm{AD}}_{k}=1-\left(1+s_{\Omega_{k}} / \bar{\Omega}_{k}\right)^{-1}$, where $\bar{\Omega}_{k}$ and $s_{\Omega_{k}}$ are the mean and the standard deviation area. The $\widehat{\mathrm{AD}}_{\mathrm{k}}$ estimate avoids the limitations seen in $\overline{\mathrm{AD}}_{k}$. In practice, micrographs that contain none or less than three particles cannot provide accurate Delaunay triangles and have to be estimated. For an upper-bound estimate $\left(\widehat{\mathrm{AD}}_{\mathrm{k}}(\mathrm{ub})\right)$, two triangles are assumed to be present that occupy half the area of the micrograph. For a lower-bound estimate $\left(\widehat{\mathrm{AD}}_{\mathrm{k}}(\mathrm{lb})\right)$, these micrographs are ignored completely.

This method is similar to that obtained through tiling or stitching micrographs together to form a larger image, thereby increasing the number of particles analysed, but is easier to perform as micrographs can and should be taken from separate regions of the material rather than an adjoining patch (which lessens the risk of being in an atypical and/or locally similar region of the material). $\widehat{A D}_{k}$ improvements are best seen in poorly dispersed systems. Revisiting the silica-rubber particle modified composite, we find that the correct type of dispersion is interpreted for the highest magnifications $(L=1,2.5$ or $3.5 \mu \mathrm{m})$ where $\overline{\mathrm{AD}}_{k}$ was misleading. This is shown in Fig. 10 by the envelope of likely measurements bounded by the two dashed lines which demonstrate that $\widehat{Z}_{k}($ upp $)>2$. The value of $\widehat{\mathrm{AD}}_{k}$ can also be seen to be much more stable with $L$, compared to $\overline{\mathrm{AD}}_{k}$, with a maximum variation of 0.2 and 0.7 , respectively. For well-dispersed systems, little benefit is brought using $\widehat{\mathrm{AD}}_{k}$ because the variation in particle number, between micrographs, is minor compared to the mean value. Hence, the dispersion type is interpreted the same and $\widehat{\mathrm{AD}}_{\mathrm{k}}$ is found to measure no more than 0.03 greater than $\overline{\mathrm{AD}}_{k}$.

Additional dispersion measurements for two species particle modified composites

For dual-modified composites, the dispersion quality of either or all types of particles may be responsible for the unique materials characteristics seen. The method of measuring Area Disorder is sufficiently versatile that it can be adapted, without reinterpretation, to describe a variety of types of particle dispersions. Two such possibilities are outlined below, describing rubber particles and nanoparticle clusters.

\section{Dispersion of rubber microparticles}

The same method of analysis can be used to measure the dispersion quality of the rubber particles. A prerequisite 
before analysis is that the contrast of the micrograph is inverted to make the darker regions of the rubber particles light and conversely the silica nanoparticles dark. The radius of the rubber particles used in the silica-rubber particle modified composite is of the order of $500 \mathrm{~nm}$, and the area fraction is given as 0.106 . Due to their macroscopic size, the diamond knife, during sample preparation, splits the rubber particle in two and means that the AFM micrographs show all the locations of the rubber along the plane. Similarly, the rubber particles' relatively large size means that they are less likely to be missed by the computer detection process and so do not require the additional subdivision of potential particles nor require adjustment to the observed area fraction. Thus, the measured value of the Area Disorder reflects the actual dispersion of the rubber and the $z$-test reverts to the perfect case given in Eq. (3) with test statistic $Z_{k}$.

Figure 11 shows an example micrograph of the silicarubber particle modified composite with the central locations of the rubber particles, determined using the computer algorithm previously described, overlaid as points. The Area Disorder is calculated as $\mathrm{AD}_{\text {Del }}$ (rubber) $=0.3941$ with $Z_{k}=-0.4371$ and the interpretation is that the dispersion of rubber particles is indistinguishable from random. Caution should be taken here as the analysis is based upon the location of only 25 particles (where the workings of $Z_{k}$ does not correctly account for the number dependence of the mean value expected for such small numbers of particles). Nonetheless this is the best that can be achieved when analysing using the same set of micrographs that are suitable for measuring nanoparticles dispersion. Thus, the point is emphasised that because rubber particles are many orders of magnitude larger than the nanoparticles, then to obtain a more precise estimate for the dispersion requires taking a separate set of AFM micrographs at much lower magnification levels.

\section{Dispersion of clusters}

Sometimes there are cases where studying the statistics of the clusters are of interest. The complexity of the cluster can be reduced by removing the internal structure of nanoparticles and treating the cluster as a macroscopic particle. This would be useful to test properties that may not be dependent on the internal structure of clusters but rather the spatial arrangement of clusters. In such a case, the homogeneity or heterogeneity of the clusters themselves may make useful statistics. Studying nanoparticle dispersion masks these large-scale differences behind more dominant and populous short-ranged particle-particle spacings within a cluster.

Here, we described a method that could be used to obtain a measure of cluster dispersion using the Area Disorder. As before a micrograph of the material is used to generate the Delaunay network based upon the locations of the nanoparticles. Select the shortest edge from each Delaunay triangle and omit the remaining two edges. This will result in a skeleton network of connected pairs (see Fig. 12a). Any remaining link that has a length greater than a cut-off length $l_{\max }$ (which is chosen by finding the lowest distance bin of a 200 bin histogram of minimum Delaunay edge length with a frequency of less than five) is subsequently removed. This leaves isolated groups of connected particles which we define to be clusters. The dispersion quality of these clusters can then be analysed using the Area Disorder measure as previously described. To avoid the need to write new specialised computer code, we simplify the problem by assuming that the exact shape of the cluster is irrelevant (i.e the average shape of a cluster is isotropically circular) such that the cluster can be contracted to the centre of mass point without changing the Delaunay tessellation. The Delaunay network for the clusters is then generated from these centre of mass
Fig. 11 Measuring the Area Disorder of rubber particles: (a) the centre of the rubber particles are picked out as points by the computer algorithm and (b) the corresponding Delaunay network
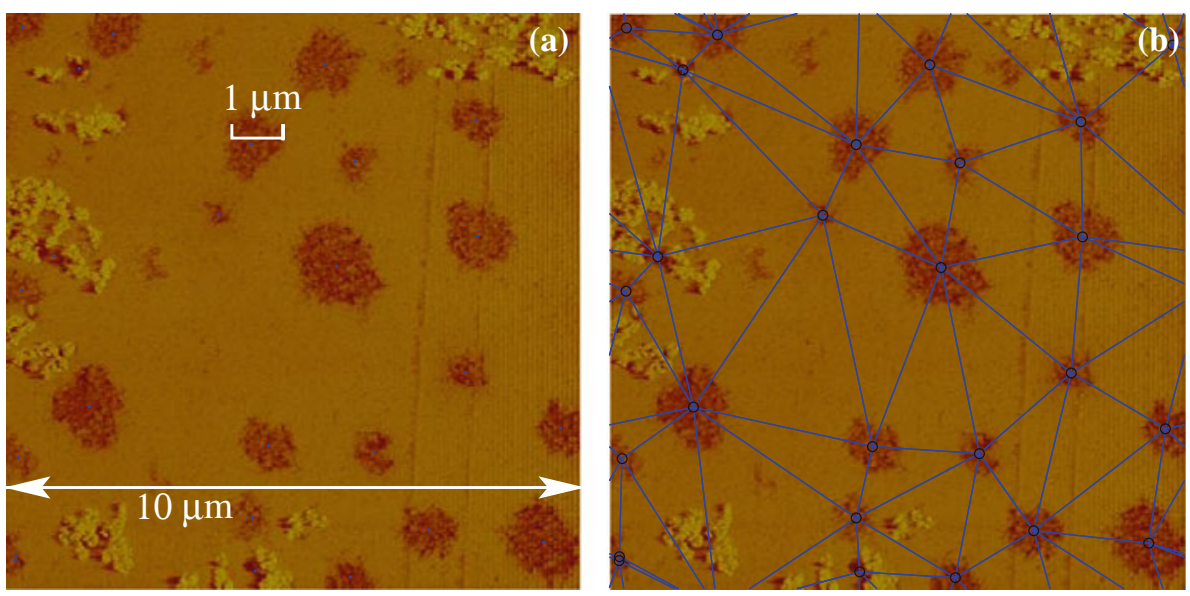
Fig. 12 Demonstration of finding the centre of mass points of clusters and generating from them a Delaunay network:

(a) shows the skeleton network of connected pairs in each cluster; (b) gives the centre of mass positions of the clusters and the generated Delaunay network
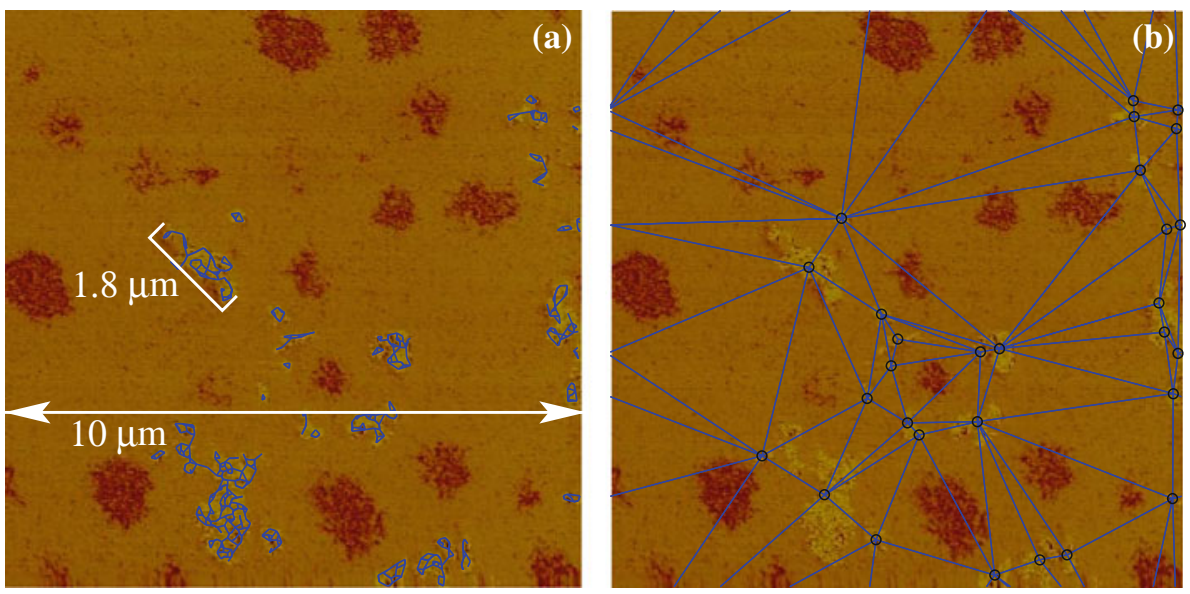

positions and used to calculate the Area Disorder for clusters (see Fig. 12b). In principle, interpretation of the Area Disorder of clusters would be the same as that for the individual nanoparticles.

For the example shown in Fig. 12, the Area Disorder of nanoparticles is read as $\mathrm{AD}_{\text {Del }}(\mathrm{Nano})=0.8363$ whereas the dispersion of the clusters is less poorly dispersed with an Area Disorder of $\mathrm{AD}_{\mathrm{Del}}$ (Clus) $=0.5350$.

\section{Conclusions}

This article has outlined some of the practices required to implement a dispersion parameter to quantify real data. By being aware of the micrographs' subjective limitations it is possible to account for the underlying data quality when evaluating the dispersion by use of an appropriate likelihood test such as the z-test used here.

The first step is to define a parameter, in this case the Area Disorder $\left(\mathrm{AD}_{\mathrm{Del}}\right)$. The behaviour for the 'perfect' data set (no errors in particle position and all particles observable) has been outlined. Regions on the diagram of $\mathrm{AD}_{\mathrm{Del}}$ versus the area fraction of nanoparticles correspond to good and bad dispersion, and the line of division between the two is at isotropic random dispersion. The second step is to allow for the natural variation in observation expected to occur between finite-sized micrographs. The inherent uncertainty in the observed value of $\mathrm{AD}_{\mathrm{Del}}$ causes the boundary between good and bad dispersion to be ill-defined. A $z$-test allows the experimenter to judge the likelihood of a sample micrograph representing a randomly dispersed material and hence upon rejection of the null hypothesis whether the material is dispersed well (better than random) or poorly.

The third step requires the experimenter to recognise that the data are likely to be imperfect. Incompleteness of particles is a problem inherent to AFM images. The fraction of particles visible in the micrograph can strongly change the apparent dispersion behaviour as their positions are influenced by (i.e not independent of) the hidden/ missing particles. Consequently: (a) a hard-core randomly dispersed system can look clustered beyond that accountable from sample variation alone; (b) the area fraction used for the RHM to compare with the experimental data is important when deciding on the strength of evidence there is for a system to be well dispersed.

A conservative approach to classifying systems involves performing either or both of the two variants of the $z$-test ( $Z_{k}$ (upp), $Z_{k}$ (low)) on the material. $Z_{k}$ (upp) is based on comparing the material against a system exhibiting complete spatial randomness (a RHM with point-particles such that $A_{f}^{\prime}=0$ ). If $Z_{k}$ (upp) is greater than 2 , then the material is highly likely to be heterogeneous and poorly dispersed. $Z_{k}$ (low) is based on comparing the material against the RHM with $A_{f}^{\prime}$ related to the observed particles. When $Z_{k}$ (low) $<-2$, then the material is highly likely to be better-dispersed than random. If neither criterion is satisfied, then the systems are indeterminate from randomly dispersed (which does not discount them from being better dispersed but we cannot tell from the data available).

In high area fraction materials $\left(A_{f} \gg 0.2\right)$ it is possible to further limit the types of materials that fall into the indeterminate class by redefining $Z_{k}$ (low) to be a test against the RHM that uses the material's known area fraction and then removes the correct fraction of particles from the data set of particles' centre of mass positions. Although this gives better realism it would involve the simulation of each specific system in the absence of known solutions.

When almost all the particles are observable then it is sufficient to perform the original $z$-test.

The choice of magnification level for the micrograph that is required for the reliable estimate of dispersion is dependent on the type of structure present (i.e whether there are individual nanoparticles or collective nano-clusters). When nanoparticles are singular, such that we suspect that good dispersion is present, then micrographs with magnifications of $2.5 \leq L \leq 5 \mu \mathrm{m}$ should be chosen to 
ensure good visibility of particles. Alternatively, if it is suspected that the system is clustered then a much larger $L$ should be used, sufficient to ensure clusters are always seen in the micrograph, and the pretence of identifying all the particles can be abandoned. When it is possible for the user to analyse the micrographs collectively, then the ensemble average of $\mathrm{AD}_{\mathrm{Del}}$ may provide a more reliable measure than $\overline{\mathrm{AD}}_{\mathrm{Del}}$. In applying these findings more generally, it should be noted that the acceptable magnification levels, given by $L$, will need to be adjusted proportionally to the radius of the nanoparticle used.

Acknowledgements The authors would like to thank the EPSRC for providing research funding under the grant EP/H00582X and Nanoresins for supplying materials.

\section{References}

1. Song YS, Youn JR (2005) Carbon 43:1378. doi:10.1016/ j.carbon.2005.01.007

2. Kinloch AJ, Taylor AC (2006) J Mater Sci 41:3271. doi:10.1007/ s10853-005-5472-0

3. Pavlidou S, Papaspyrides CD (2008) Prog Polym Sci 33:1119. doi:10.1016/j.progpolymsci.2008.07.008

4. Paul DR, Robeson LM (2008) Polymer 49:3187. doi: 10.1016/j.polymer.2008.04.017

5. Manjunatha CM, Taylor AC, Kinloch AJ, Sprenger S (2009) J Mater Sci 44:4487. doi:10.1007/s10853-009-3653-y

6. Chen CH, Jian JY, Yen FS (2009) Composites Part A-Appl S 40:463. doi:10.1016/j.compositesa.2009.01.010

7. Gershon A, Cole D, Kota A, Bruck H (2010) J Mater Sci 45:6353. doi:10.1007/s10853-010-4597-y
8. Luo ZP (2010) J Mater Sci 45:3228. doi:10.1007/ s10853-010-4330-x

9. Bakshi SR, Batista RG, Agarwal A (2009) Composites Part A-Appl S 40:1311. doi:10.1016/j.compositesa.2009.06.004

10. Berndt S, Bretschneider J, Helm H, Stoyan D (1996) Mater Charact 36:93

11. Davy PJ, Guild FJ (1988) P Roy Soc Lond A Mat 418:95

12. Ghosh S, Nowak Z, Lee K (1997) Acta Mater 45:2215

13. Li M, Ghosh S, Richmond O, Weiland H, Rouns TN (1999) Mater Sci Eng A 265:153

14. Heijman MJGW, Benes NE, ten Elshof JE, Verweij H (2002) Mater Res Bull 37:141

15. Hendriks MGHM, Heijman MJGW, van Zyl WE, ten Elshof JE, Verweij H (2002) J Am Ceram Soc 85:2097

16. Al-Ostaz A, Diwakar A, Alzebdeh KI (2007) J Mater Sci 42:7016. doi:10.1007/s10853-006-1117-1

17. Zhu Y, Allen GC, Adams JM, Gittins D, Heard PJ, Skuse DR (2010) Compos Struct 92:2203. doi:10.1016/j.compstruct.2009. 08.045

18. Khare HS, Burris DL (2010) Polymer 51:719. doi:10.1016/ j.polymer.2009.12.031

19. Yazdanbakhsh A, Grasley Z, Tyson B, Abu Al-Rub RK (2011) Composites Part A-Appl S 42:75. doi:10.1016/j.physletb.2003. 10.071

20. Bray DJ, Gilmour SG, Guild FJ, Taylor AC (2010) In: Proceedings of the 3rd international conference of the ERCIM working group on computing \& statistics. Elsevier, London, p 29

21. Bray DJ, Gilmour SG, Guild FJ, Taylor AC (2011) J Roy Stat Soc C-App. Submitted

22. Marcelpoil R, Usson Y (1992) J Theor Biol 154:359

23. Johnsen BB, Kinloch AJ, Mohammed RD, Taylor AC, Sprenger S (2007) Polymer 48:530. doi:10.1016/j.polymer.2006.11.038

24. Hsieh TH, Kinloch AJ, Masania K, Sohn Lee J, Taylor AC, Sprenger S (2010) J Mater Sci 45:1193. doi:10.1007/s10853009-4064-9

25. Dias A, Buono V, Vilela J, Andrade M, Lima T (1997) J Mater Sci 32:4715. doi:10.1023/A:1018618628027 